\title{
Earthquake induced landslide susceptibility mapping using an integrated ensemble frequency ratio and logistic regression models in West Sumatera Province, Indonesia
}

\begin{abstract}
An 8 Richter Scale (RS) earthquake struck West Sumatra on Wednesday, 30 September 2009 , at $17.16 \mathrm{pm}$ which led to huge number of landslides. Hence a comprehensive landslide susceptibility mapping (LSM) should be produced in order to reduce the damages to people and infrastructures. In the international landslide literature, various statistical methods such as frequency ratio (FR) and logistic regression (LR) have been widely used individually for LSM, but they have some weaknesses. FR which is able to perform bivariate statistical analysis (BSA) assesses the influence of classes of each conditioning factor on landslide occurrence. However, the correlation between the factors is mostly neglected. On the other hand, LR is able to analyze the relationship among the factors while it is not capable to evaluate the classes of each landslide conditioning factor. This paper aims to propose an ensemble method of FR and LR in order to overcome their weak points. For LSM, a landslide inventory map with a total of 87 landslide locations was extracted from various sources. Then the landslide inventory was randomly divided into two datasets $70 \%$ for training the models and the remaining $30 \%$ was used for validation purpose. The landslide conditioning factors consist of: altitude, curvature, river, SPI, rainfall, soil type, soil texture, land use/cover (LULC), peak ground acceleration (PGA), geology, slope, aspect, lineament and topographic wetness index (TWI). Four PGA of 7.5, 8, 8.6 and 9 were acquired and PGA 8 which was related to the 2009 earthquake was used to generate the model. Finally, the produced landslide susceptibility maps were validated using an area under the (ROC) curve method. For the model which was derived by PGA 8 , the validation results showed $84 \%$ and $78 \%$ success and prediction rates respectively. Furthermore, the prediction rates for the models made by PGA $7.2,8.6$ and 9 are $79 \%, 78 \%$ and $81 \%$ respectively. The result proved the reasonable efficiency of the proposed method for earthquake induced landslide susceptibility mapping. Also the proposed ensemble method can be used in other hazard studies as it is capable to produce rapid and accurate assessment for disaster management and decision making.
\end{abstract}

Keyword: Landslide; Earthquake; Ensemble; Geographic information system (GIS); Remote sensing (RS); Indonesia 